\title{
On the liquid drop model mass formulae and charge radii
}

\author{
G. Royer and R. Rousseau
}

Laboratoire Subatech, UMR : IN2P3/CNRS-Université-Ecole des Mines, 44 Nantes, France

Received: date / Revised version: date

\begin{abstract}
An adjustment to 782 ground state nuclear charge radii for nuclei with $\mathrm{N}, \mathrm{Z} \geq 8$ leads to $R_{0}=1.2257 A^{1 / 3} \mathrm{fm}$ and $\sigma=0.124 \mathrm{fm}$ for the charge radius. Assuming such a Coulomb energy $E_{c}=\frac{3}{5} e^{2} Z^{2} / 1.2257 A^{\frac{1}{3}}$, the coefficients of different possible mass formulae derived from the liquid drop model and including the shell and pairing energies have been determined from 2027 masses verifying N,Z $\geq 8$ and a mass uncertainty $\leq 150 \mathrm{keV}$. These formulae take into account or not the diffuseness correction $\left(Z^{2} / A\right.$ term), the charge exchange correction term $\left(Z^{4 / 3} / A^{1 / 3}\right.$ term), the curvature energy, the Wigner terms and different powers of $I=(N-Z) / A$. The Coulomb diffuseness correction or the charge exchange correction term plays the main role to improve the accuracy of the mass formulae. The different fits lead to a surface energy coefficient of around $17-18 \mathrm{MeV}$. A possible more precise formula for the Coulomb radius is $R_{0}=1.2332 A^{1 / 3}+2.8961 / A^{2 / 3}-0.18688 A^{1 / 3} I \mathrm{fm}$ with $\sigma=0.052 \mathrm{fm}$.
\end{abstract}

PACS. 21.10.Dr Binding energies and masses - 21.60.Ev Collective models

\section{Introduction}

The prediction of the binding energies of new exotic nuclei both in the regions close to the proton and neutron drip lines and in the superheavy element region requires very accurate mass formulae. Historically, within a modelling of the nucleus by a charged liquid drop, semimacroscopic models including a pairing energy have been firstly developed to reproduce the experimental nuclear masses [1,2]. Later on, macroscopic-microscopic approaches have been developed, mainly the finite-range liquid drop model and the finite-range droplet model [3]. They take into account the non-smooth values of the masses (due to the shell effects and other microscopic properties) and the deformation of the nuclei investigating the fission and alpha emission exit channels or the fusion entrance channels. Nuclear masses have also been obtained accurately within a recent version of the statistical Thomas-Fermi model [4, 5]. More recently, microscopic Hartree-Fock self-consistent theories using mean-fields and Skyrme or Gogny forces and pairing correlations $[6,7]$ as well as relativistic mean field theories [8] have also been used to reproduce these nuclear masses.

In the macroscopic-microscopic liquid drop model approaches the evolution of the nucleus is mainly governed by the balance between the effects of the repulsive Coulomb forces and attractive surface tension and proximity forces [9-11]. In these approaches the charge radius is simply given by $R_{0}=r_{0} A^{1 / 3}$ and $r_{0}$ varies essentially from 1.12 to $1.24 \mathrm{fm}$ according to the models. In the second section a value of $r_{0}$ is extracted from a whole set of recent experimental data. Later on, different possible additional terms entering into the mass formulae derived from the liquid drop model are briefly detailed in section 3. The coefficients of the liquid drop model development have been determined in section 4 using the value of $r_{0}$ extracted from experiments to calculate the Coulomb energy. All the studied mass formulae contain the usual volume, surface, Coulomb energy terms and the shell and pairing energies and include or not the diffuseness correction to the Coulomb energy, the charge exchange correction term, the curvature energy, a constant term, different forms of the Wigner term and different powers of the relative neutron excess $I=(N-Z) / A$.

\section{Nuclear charge radii}

For nuclei with $\mathrm{N}, \mathrm{Z} \geq 8$ the experimental set of 782 ground state nuclear charge radii presented in ref. [12] leads to a rms charge radius of $0.94944 A^{1 / 3}$ which gives for the charge radius defined as

$$
R_{0}=\sqrt{\frac{5}{3}}<r^{2}>^{1 / 2}
$$

the value $R_{0}=1.2257 A^{1 / 3} \mathrm{fm}$ with $\sigma=0.124 \mathrm{fm}$. It is worth-while to mention that the experimental data show that the ratio $R_{0} / A^{1 / 3}$ is far from being constant. For example, $R_{0} / A^{1 / 3}=1.312 \mathrm{fm}$ for ${ }^{40} \mathrm{Ca}$ and $R_{0} / A^{1 / 3}=1.234$ for ${ }^{48} \mathrm{Ca}$ while $R_{0} / A^{1 / 3}=1.217$ for ${ }^{190} \mathrm{~Pb}$ and $R_{0} / A^{1 / 3}=$ $1.201 \mathrm{fm}$ for ${ }^{214} \mathrm{~Pb}$. In a preceding paper [13] several nuclear mass formulae have been determined leaving the parameter $r_{0}$ free to evolve. It is quite satisfying to observe 
that $\mathrm{r}_{0}$ converges to $1.22-1.23 \mathrm{fm}$ in good agreement with the experimental data for the charge radius.

More precise formulae allow to better reproduce the experimental charge radius. For example, the expression proposed in Ref. [14] has been fitted again and the new formula

$$
R_{0}=1.2332 A^{1 / 3}+\frac{2.8961}{A^{2 / 3}}-0.18688 A^{1 / 3} I \mathrm{fm}
$$

gives a good accuracy $(\sigma=0.052 \mathrm{fm})$. The introduction of the ground state deformation via a promiscuity factor depending on the distance of $\mathrm{Z}$ an $\mathrm{N}$ to the proton and neutron magic numbers can still improve the precision [15].

Nevertheless, the introduction in the mass formulae of such expressions to calculate the Coulomb energy does not allow to improve the accuracy of the mass formulae since the liquid drop model mass formulae basically suppose the proportionality of the charge and mass radius to $A^{1 / 3}$.

\section{Nuclear binding energy}

The nuclear mass is directly connected to the nuclear binding energy $\mathrm{B}$ and several subsets of the following expansion of the nuclear binding energy in powers of $A^{-1 / 3}$ and $|I|$ have been investigated :

$$
\begin{array}{r}
B=a_{v}\left(1-k_{v_{1}}|I|-k_{v_{2}} I^{2}\right) A \\
-a_{s}\left(1-k_{s_{1}}|I|-k_{s_{2}} I^{2}\right) A^{\frac{2}{3}} \\
-a_{k}\left(1-k_{k_{1}}|I|-k_{k_{2}} I^{2}\right) A^{\frac{1}{3}} \\
-a_{0} A^{0}-\frac{3}{5} \frac{e^{2} Z^{2}}{1.2257 A^{\frac{1}{3}}}+f_{p} \frac{Z^{2}}{A} \\
+a_{c, \text { exc }} \frac{Z^{\frac{4}{3}}}{A^{\frac{1}{3}}}-E_{\text {pairing }}-E_{\text {shell }}-E_{\text {Wigner }} .
\end{array}
$$

The first term is the volume energy in the infinite nuclear matter. $I^{2} A$ is the asymmetry energy term of the BetheWeizsäcker mass formula. The second term gives the surface energy and expresses the deficit of binding energy of the nucleons at the nuclear surface. The Bethe-Weizsäcker mass formula does not take into account the dependence of the surface energy on $I$. The following term is the curvature energy which appears to correct the surface energy when the local curvature properties are considered. This term is not taken into account in the FRLDM [3] but appears in the Lublin-Strasbourg Drop (LSD) model [16] and the TF model [5]. In the three first terms a dependence on $|I|$ has been introduced since they have been proposed to better reproduce the fission barrier heights [17]. The $\mathrm{A}^{0}$ term appears when the surface term is developed to include higher order terms in $\mathrm{A}^{-1 / 3}$ and $I$. The fifth term gives the decrease of binding energy due to the repulsion between the protons. The charge radius is fixed to the value derived from the recent experimental data. This Coulomb energy is proportional to $Z(Z-1)$ in the Bethe-Weizsäcker mass formula. The $Z^{2} / A$ term is the diffuseness correction to the basic sharp radius Coulomb energy term (called also the proton form-factor correction to the Coulomb energy in Ref. [3]) and the term proportional to $Z^{4 / 3} / A^{1 / 3}$ is the charge exchange correction term.

The pairing energy has been calculated within the formulae proposed in the recent version of the Thomas-Fermi model [5]. The theoretical shell effects used in the TF model ( $7^{\text {th }}$ column of the table in [4] and [5]) have also been used since they have allowed to reproduce correctly the masses from fermium to $Z=112$. They have been derived from the Strutinsky shell-correction method. As an example, they give a contribution of $12.84 \mathrm{MeV}$ to the binding energy of ${ }^{208} \mathrm{~Pb}$. The fits on nuclear masses depend necessarily on the choice of the selected theoretical shell effects. The sign for the shell energy term comes from the adopted definition in [4].

The Wigner energy has been introduced to reproduce the kink in the nuclear mass surface. It is not a usual shell effect. It appears in the counting of identical pairs in a nucleus and depends on $I$. The original expression is simply $W|I|[18]$. Its effect is to decrease the binding energy when $N \neq Z$.

The congruence energy term is expressed as :

$$
E_{\text {cong }}=-10 \exp (-4.2|I|) \mathrm{MeV} .
$$

It is an extra binding energy associated also with the presence of congruent pairs [5].

Within an Hartree-Fock approach [19] it has been argued that a gaussian expression

$$
E=V_{W} \exp \left(-\lambda I^{2}\right)
$$

is as acceptable.

Another term has also been proposed in Ref. [19]

$$
E=\beta|N-Z| \exp \left[-\left(A / A_{0}\right)^{2}\right] .
$$

Another expression has also been tested

$$
E=\beta|N-Z| \exp \left[-\left(A / A_{0}\right)\right]
$$

\section{Coefficients of the mass formulae}

The coefficients of different expansions in powers of $A^{-1 / 3}$ and $|I|$ have been obtained by a least square fitting procedure to 2027 nuclear masses verifying $\mathrm{N}, \mathrm{Z} \geq 8$ and a mass uncertainty $\leq 150 \mathrm{keV}[20]$. To compare the relative efficiency of the different selected sets of terms the rootmean-square deviation $\sigma$ defined by

$$
\sigma^{2}=\frac{\Sigma\left[M_{T h}-M_{E x p}\right]^{2}}{n}
$$

has been used.

In the Table I, the improvement of the nuclear mass reproduction according to the introduction of different additional terms is displayed. The basic A, $A I^{2}, A^{2 / 3}, A^{2 / 3} I^{2}$ and $Z^{2} / 1.2257 A^{1 / 3}$ terms lead already to a deviation of only $0.99 \mathrm{MeV}$.

The diffuseness correction term (in $Z^{2} / A$ ) as well as the charge exchange correction term (in $Z^{4 / 3} / A^{1 / 3}$ ) are 
very efficient to reduce the deviation in the same order of magnitude and towards $\sigma=0.67 \mathrm{MeV}$. When both the diffuseness correction to the Coulomb energy and the charge exchange correction term are taken into account the deviation remains the same and spurious coefficients appear. The introduction of a constant term is useless and can induce a discontinuity during the transition from one to twobody shapes as in fission, fusion or $\alpha$ emission. The first expression of the Wigner term $\mathrm{W}|I|$ allows to diminish the error of only $0.13 \mathrm{MeV}$. Its main drawback is that it is approximately independent of the nuclear shape [18]. Then, in a division into 2 fragments, both with the same value of $|I|$, the Wigner energy jumps at scission to 2 times its original value leading, for an example, to a discontinuity of the potential energy of around $6.7 \mathrm{MeV}$ at the contact point between spherical fragments of ${ }^{258} \mathrm{Fm}$. The influence of the congruence term is very weak at least with the fixed coefficients adopted here. When the coefficients before the exponential and the exponent are free the congruence energy tends to the usual Wigner term since the coefficient before the exponential diminishes while the exponent increases. The congruence energy term leads also to an important discontinuity : $3.9 \mathrm{MeV}$ for ${ }^{258} \mathrm{Fm}$. The Coulomb diffuseness correction term has the main advantage to be almost continuous at the scission point in the entrance or exit channels. The introduction of the curvature energy in $A^{1 / 3}$ alone does not allow to significantly decrease the deviation from the experimental masses but when both the terms in $A^{1 / 3}$ and $A^{1 / 3} I^{2}$ are taken into account $\sigma$ is reduced to $0.64 \mathrm{MeV}$. These terms have the advantage to be almost continuous in a symmetric decay but their values and signs lack of stability. The values of $A_{0}$ which minimize the mass rms deviation when the formulae (6) and (7) are introduced are respectively 48 and 35 for the two expressions. These formulae for the Wigner energy are supposed to be approximately independent of the nuclear shape. Their discontinuity at the scission point of fission or fusion barriers is less important than that of the congruence and $\mathrm{W}|I|$ terms. For example, for the expressions $|N-Z| \exp \left[-(A / 48)^{2}\right]$ and $1.5|N-Z| \exp [-(A / 35)]$ and symmetric decay of the ${ }^{258} \mathrm{Fm}$ the discontinuities at the contact point of the nascent fragments are respectively 0 and $0.05 \mathrm{MeV}$. The introduction of an $|I|$ dependence in the volume and surface energy terms improves the accuracy by $0.03-0.04 \mathrm{MeV}$. When both the Coulomb diffuseness correction term and the pure Wigner term are taken into account the deviation diminishes till $\sigma=0.61 \mathrm{MeV}$. The lowest value which can be attained when adding other terms seems to be $0.58 \mathrm{MeV}$. With only 6 adjustable parameters different combinations lead to $\sigma=0.61 \mathrm{MeV}$. Recently, the mutual influence of terms in semi-empirical formulae has been deeply investigated [21].

As an example, Fig. 1 indicates the difference between the theoretical and experimental masses using the formula

$$
\begin{aligned}
& B=15.3543\left(1-1.7445 I^{2}\right) A \\
& -17.2293\left(1-1.5765 I^{2}\right) A^{2 / 3} \\
& \quad-\frac{0.6 e^{2} Z^{2}}{1.2257 A^{1 / 3}}+1.24413 \frac{Z^{2}}{A}-E_{\text {pair }}
\end{aligned}
$$

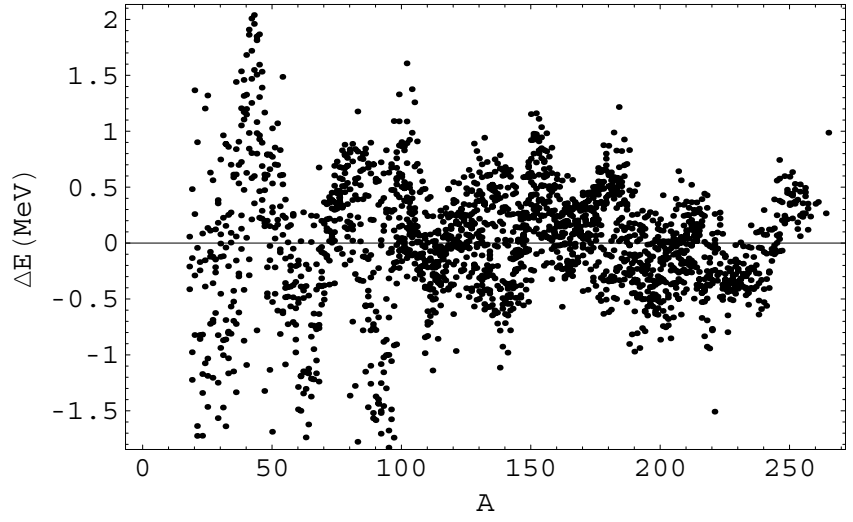

Fig. 1. Difference between the theoretical and experimental nuclear binding energies for the 2027 selected nuclei versus the mass number.

$$
-E_{\text {shell }}-0.7614|N-Z| e^{-(A / 35)}
$$

leading to $\sigma=0.61 \mathrm{MeV}$. For most of the nuclei with A $\geq 110$ the difference is less than $1 \mathrm{MeV}$ and it appears no strong structure in the set of errors. For $\mathrm{A} \leq 110$ the cloud of points is less uniform and more structured. This is probably due to the values of the pairing and shell energies.

\section{Conclusion}

An adjustment to 782 recent ground state nuclear charge radii for nuclei with $\mathrm{N}, \mathrm{Z} \geq 8$ leads to $R_{0}=1.2257 A^{1 / 3} \mathrm{fm}$. This value is close to the value $R_{0}=1.2249 A^{1 / 3}$ fm proposed in [22]. Assuming such a Coulomb energy $E_{c}=$ $\frac{3}{5} e^{2} Z^{2} / 1.2257 A^{\frac{1}{3}}$, the coefficients of different possible mass formulae derived from the liquid drop model and including the shell and pairing energies have been revisited and determined from 2027 masses verifying $\mathrm{N}, \mathrm{Z} \geq 8$ and a mass uncertainty $\leq 150 \mathrm{keV}$.

The Coulomb diffuseness correction $Z^{2} / A$ term or the charge exchange correction $Z^{4 / 3} / A^{1 / 3}$ term plays the main role to improve the accuracy of the mass formulae. With a less efficiency, the Wigner term and the curvature energy can also be used separately. The introduction of an $|I|$ dependence in the surface and volume energies improves slightly the efficiency of the expansion. The pure Wigner, the congruence and the constant terms lead to an important discontinuity of the deformation energy during the transition from one to two-body shapes as in fission, fusion or $\alpha$ emission. The congruence energy term is much less efficient than the Wigner term $\mathrm{W}|I|$. Finally, the different best fits lead to a root-mean-square mass deviation of $0.58 \mathrm{MeV}$ and a surface energy coefficient of around 17$18 \mathrm{MeV}$. For comparison the values adopted in [16],[22] and [23] are respectively 16.9707, 17.9439 and $21.7 \mathrm{MeV}$.

\section{References}

1. C.F. von Weizsäcker, Z. Physik 96 (1935) 431. 
2. H.A. Bethe, R.F. Bacher, Rev. Mod. Phys. 8 (1936) 82.

3. P. Möller, J.R. Nix, W.D. Myers, W.J. Swiatecki, At. Data Nucl. Data Tables 59 (1995) 185.

4. W.D. Myers, W.J. Swiatecki, LBL report 36803, 1994.

5. W.D. Myers, W.J. Swiatecki, Nucl. Phys. A 601 (1996) 141.

6. M. Samyn, S. Goriely, P.-H. Heenen, J.M. Pearson, F. Tondeur, Nucl. Phys. A 700 (2002) 142.

7. J. Rikovska Stone, J. Phys. G 31 (2005) R211.

8. M. Bender, et al., Phys. Lett. B 515 (2001) 42.

9. G. Royer, B. Remaud, J. Phys. G 10 (1984) 1057.

10. G. Royer, B. Remaud, Nucl. Phys. A 444 (1985) 477.

11. G. Royer, J. Phys. G 26 (2000) 1149.

12. I. Angeli, At. Data Nucl. Data Tables 87 (2004) 185.

13. G. Royer, Nucl. Phys. A 807 (2008) 105.

14. W.D. Myers, W.J. Swiatecki, Phys. Rev. C 62 (2000) 044610.

15. J. Duflo, A.P. Zucker, Phys. Rev. C 66 (2002) 051304(R).

16. K. Pomorski, J. Dudek, Phys. Rev. C 67 (2003) 044316.

17. M. Dahlinger, D. Vermeulen, K.H. Schmidt, Nucl. Phys. A 376 (1982) 94.

18. W.D. Myers, Droplet Model of Atomic Nuclei, Plenum, New York, 1977.

19. S. Goriely, M. Samyn, P.-H. Heenen, J.M. Pearson, F. Tondeur, Phys. Rev. C 66 (2002) 024326.

20. G. Audi, A.H. Wapstra, C. Thibault, Nucl. Phys. A 729 (2003) 337.

21. M. W. Kirson, Nucl. Phys. A 798 (2008) 29.

22. W.D. Myers, W.J. Swiatecki, Arkiv för Fysik, 36 (1966) 343.

23. H.J. Krappe, J.R. Nix, A.J. Sierk, Phys. Rev. C 20 (1979) 992.
Table 1. Dependence of the energy coefficient values (in MeV) on the selected term set and root mean square deviation. The theoretical shell and pairing energies are taken into account.

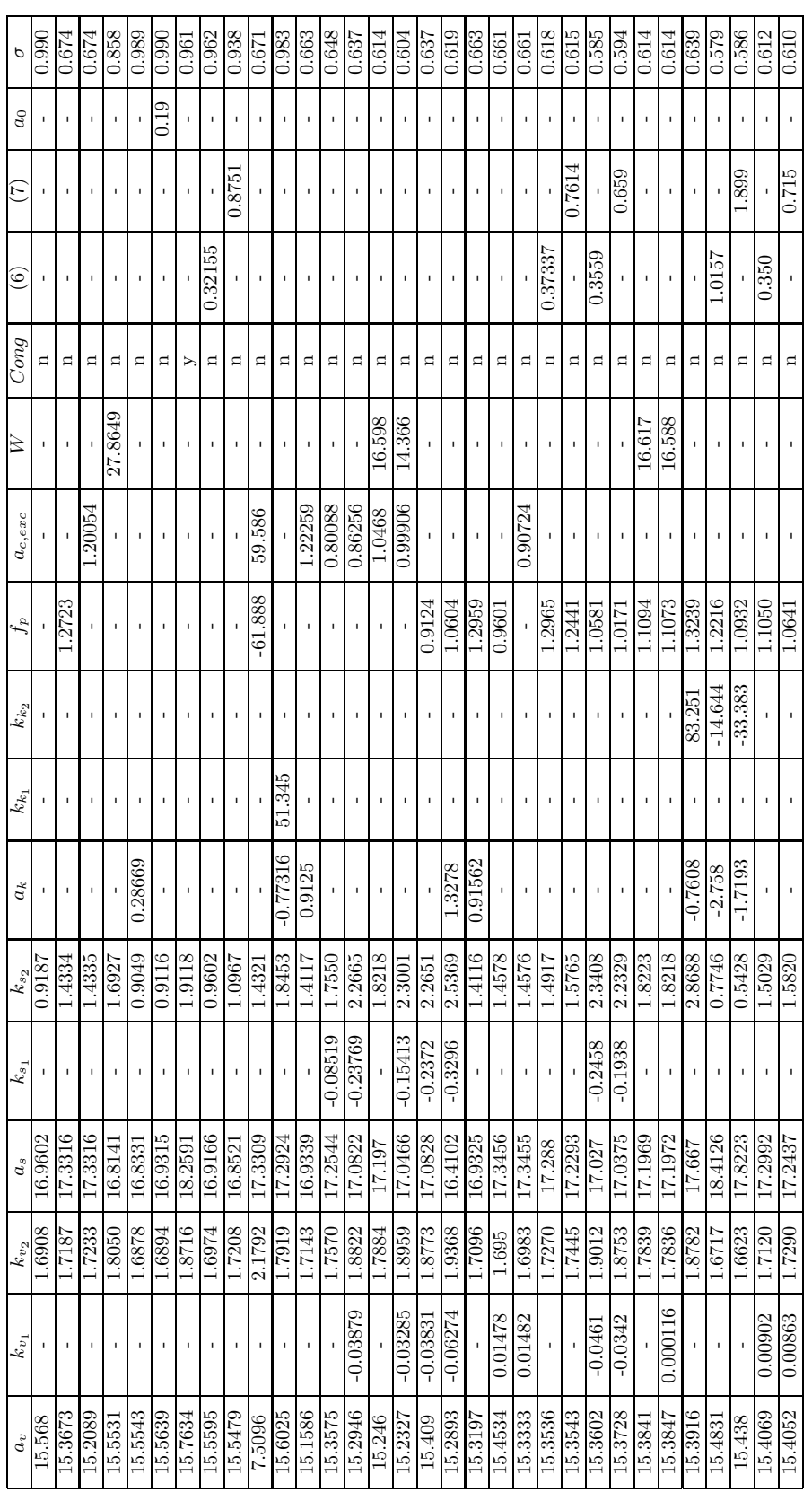

